\title{
Estereótipos de Gênero Aplicados a Mulheres Atletas
}

\author{
Gislane Ferreira de Melo ${ }^{1}$ \\ Adriana Giavoni \\ Universidade Católica de Brasília \\ Bartholomeu Torres Tróccoli \\ Universidade de Brasília
}

\begin{abstract}
RESUMO - O propósito deste estudo foi avaliar se as características do desporto e o biotipo de mulheres atletas desencadeiam a aplicação de estereótipos sexuais. Foram avaliados três grupos que diferiam quanto ao envolvimento com o desporto: a) grupo 1 - formado por atletas, b) grupo 2 - formado por alunos e profissionais de Educação Física e c) grupo 3 - formado por sedentários. O instrumento utilizado foi o Inventário dos Esquemas de Gênero do Autoconceito (IEGA). Análises de variância multivariada (One-Way MANOVA) foram realizadas correlacionando a variável independente "grupo" com os fatores das escalas masculina e feminina (variáveis dependentes) do instrumento. Os resultados demonstraram que os grupos não diferem em relação aos fatores da escala masculina, mas o grupo 3 difere dos demais grupos em relação aos fatores Sensualidade e Responsabilidade da escala feminina. Conclui-se que as características do desporto somadas e o biotipo das atletas contribuem para a aplicação de estereótipos sexuais.
\end{abstract}

Palavras-chave: estereótipos; masculinidade; feminilidade; esporte.

\section{Gender Stereotypes Applied in Female Athletes}

\begin{abstract}
The purpose of this article was to evaluate if the sport characteristics and athlete biotype promote sex stereotypes applications. Three groups with different sport involvement were analyzed: a) group 1 - compounded by athletes, b) group 2 - compounded by Physical Educators (students and teachers) and c) group 3 - compounded by sedentary people. The SelfConcept Gender Schemas Inventory (IEGA) was used to evaluate the sample. One Way Multivariate Analysis of Variance (MANOVA) were performed among "groups" (independent variable) and masculine and feminine factors (dependent variables) of the instrument. The results showed that there were no differences among groups in masculine factors, but there were in feminine factors Sensuality and Responsibility. The sedentary people perceived female athletes as having fewer feminine characteristics than the other groups did. These results confirm the hypothesis that sport characteristics and athlete biotype promote sex stereotypes applications.
\end{abstract}

Key words: stereotype; masculinity; femininity; sports.

Etimologicamente, o termo estereótipo deriva de duas palavras gregas stereo (rígido) e tipo (traço), e refere-se a "tornar fixo, inalterável" (Ferreira, 1999). Aplicado à Psicologia, este termo designa um sistema de crenças compartilhadas acerca de atributos, geralmente traços de personalidade ou comportamentos costumeiros, atribuídos a determinados grupos (Rodrigues, Assmar \& Jablonski, 1999). É uma construção cognitiva ou sócio-cognitiva a respeito de características compartilhadas por determinados grupos. Enquanto construção cognitiva, os estereótipos assemelham-se aos esquemas cognitivos que são estruturas formadas a partir de nossas experiências passadas, compostas por uma associação de variáveis que permitem aos indivíduos compreenderem as suas próprias experiências e a organizar a ampla variedade de informações que possuem sobre si mesmos e sobre os outros (Markus, 1977; Markus, Crane, Bernstein \& Siladi, 1982).

A psicologia cognitiva argumenta que os seres humanos, diante da quantidade de informação a que estão submetidos, tendem

1 Endereço: QS 07 Rua 800 lote 01 Bloco A ap. 203, Águas Claras, Brasília, DF, Brasil 70000-000. E-mail:gmelo@pos.ucb.br a agrupar os objetos em categorias, que permitem um melhor controle ambiental e uma interação mais eficaz com o meio. Uma das conseqüencias desta reconceptualização afeta a interpretação e o valor designado aos estereótipos, que passam de simples preconceito social a serem descritos como categorias, potencialmente neutras, que operam de forma similar a qualquer outro esquema. (Barberá, 1998, p. 91)

Dessa maneira, os estereótipos assim como os esquemas cognitivos funcionam como lentes que filtram as informações, retendo, organizando e estruturando somente estímulos considerados concernentes à estrutura cognitiva. "O resultado desta organização é um padrão perceptivo, o qual será utilizado como base para futuros julgamentos, decisões, inferências e predições sobre o self' (Markus, 1977, p. 64) e sobre os outros.

Sendo uma imagem mental de alta elaboração cognitiva, o termo estereótipo abarca, portanto, duas idéias centrais:

a) a conceptualização de estereótipo enquanto construção subjetiva que inclui crenças, expectativas, atribuições causais, o que significa que os estereótipos não coincidem com a realidade e b) um estereótipo não está composto por um único pensamento 
ou idéia singular senão por um conjunto organizado de idéias que se acoplam entre si. (Barberá, 1998, p. 85)

Dentre os estudos elaborados envolvendo o conceito de estereótipo encontram-se aqueles que relacionam os traços de personalidade com gênero, ou seja, os estereótipos de gênero. Nestes estudos, os traços de personalidade são agrupados em dois grandes grupos segundo a similaridade do traço com a construção sócio-cultural dos conceitos de masculinidade e feminilidade. Assim, traços individualistas ou instrumentais (por exemplo: independente, agressivo, racional) caracterizam-se como sendo pertinentes à masculinidade e traços coletivistas ou expressivos (por exemplo: amorosa, sensível, delicada) como pertinentes à feminilidade. Sendo a masculinidade e a feminilidade construtos sociais que definem características pertinentes a homens e mulheres, respectivamente.

Considerados como conceitos unidimensionais no início do século passado, por terem sido tomados como conceitos complementares e, portanto, similares à função reprodutora em que ser homem é não ser mulher e vice-versa, os conceitos de masculinidade e feminilidade assumiram uma concepção bidimensional a partir da década de 70 (Bem, 1974; Constantinople, 1973; Spence, Helmreich \& Stapp, 1975). Nesta nova concepção, a masculinidade e a feminilidade passam a ser construções psicológicas, formadas a partir das interações sócio-culturais e, portanto, independentes da polaridade biológica a que foram inseridas inicialmente. Atualmente, os conceitos de masculinidade e feminilidade são concebidos como estruturas multidimensionais (Antill \& Russell, 1982; Archer, 1989; Aubé \& Koestner, 1992; Bernard, 1981; Feather, 1978; Gaa, Liberman \& Edwards, 1979, Gaudreau, 1977; Koestner \& Aubé, 1995).

Enquanto construções sociais na qual os indivíduos encontram-se inseridos desde o nascimento, os conceitos de masculinidade e feminilidade acabam por moldar-se na subjetividade individual em estruturas semelhantes aos estereótipos de gênero, denominadas de esquemas de gênero. Subdivididos em dois esquemas distintos - o esquema masculino e o esquema feminino, os esquemas de gênero são construções subjetivas dos conceitos de masculinidade e feminilidade que se encontram presentes no autoconceito (Bem, 1981; Markus \& cols., 1982). E, assim como estes conceitos, os esquemas de gênero também apresentam composição multidimensional (Giavoni \& Tamayo, 2000).

Formado por uma associação de variáveis representativas da masculinidade, o esquema masculino filtra estímulos relacionados a este conceito, influenciando na forma de pensar, sentir e se comportar do indivíduo em relação a ele mesmo e aos outros; o mesmo ocorrendo com o esquema feminino, o qual filtrará estímulos relacionados à feminilidade. Enquanto construções psicológicas e independentes, os esquemas masculino e feminino encontram-se presentes em todos os indivíduos, variando quanto ao nível de desenvolvimento. Quando avaliados como um par, a combinação resultante dos esquemas masculino e feminino poderá variar desde pares com predomínio do esquema masculino sobre o esquema feminino a pares com predomínio do esquema feminino sobre o esquema masculino (Giavoni, 2000).
Considerando que os esquemas de gênero são construções subjetivas dos conceitos de masculinidade e feminilidade e que os indivíduos são portadores destes dois esquemas cognitivos, este trabalho visou avaliar a percepção de três grupos em relação à imagem física de mulheres atletas praticantes de desporto com predomínio de características masculinas. Isto porque os padrões culturais de masculinidade e feminilidade perpassam as mais variadas esferas, influenciando inclusive na dimensão desportiva. Em literatura encontrou-se apenas um estudo que relaciona a aplicação de estereótipos com biotipo, característica do desporto e sexo dos atletas (Giavoni, 2002). Por ser este último um estudo exploratório realizado apenas com nadadoras, procurou-se neste estudo ampliar e confirmar a extensão da aplicação de estereótipos sexuais por parte de grupos que apresentam baixo envolvimento com o desporto.

Os desportos, de uma maneira geral, podem ser classificados como possuidores de mais características masculinas (por exemplo: hóquei, futebol, halterofilismo) ou mais características femininas (por exemplo: ginástica rítmica desportiva, nado sincronizado, dança, patinação). Estas características, tomadas de uma forma inconsciente, interferem no julgamento dos indivíduos, principalmente quando o sexo do atleta contraria a característica do desporto praticado. Além da característica do desporto e do sexo do atleta, outra variável que interfere no julgamento dos indivíduos é o biotipo dos atletas, resultante da prática desportiva.

Culturalmente, há um padrão estético definido para a feminilidade e para a masculinidade. Estudos de Franzoi e Shields (1984) confirmam a importância que as mulheres atribuem à imagem física, revelando implicitamente a relação da feminilidade com o meio sócio-cultural. Neste estudo, dois dos fatores que definem a auto-estima corporal das mulheres estão associados à preocupação com a aparência física vinculada à atratividade sexual e controle do peso. Para as mulheres, a auto-estima corporal é positiva quando ela acredita que determinadas partes corporais ambicionadas pelos homens são avaliadas por elas de forma positiva, ou seja, a sua auto-estima corporal perpassa a dimensão do outro, mesmo que de uma forma projetiva.

Em seus estudos Franzoi (1995) e Franzoi, Kessenich e Surgrue (1989) revelam que a masculinidade apresenta correlação positivamente com a auto-estima, o mesmo não ocorrendo com a feminilidade. Os autores demonstram que mulheres femininas apresentam maior dificuldade em liberar-se dos padrões culturais definidos para a feminilidade, padrões estes que definem o corpo feminino como um "ornamento de beleza" (Franzoi, 1995, p. 421). Andersen (2000) descreve que o Instituto Australiano de Esportes demonstrou que mulheres atletas vivenciam estados de ansiedade em relação às suas imagens corporais, devido "à pressão social que estabelece um tipo diferente de corpo, consistente com os padrões de gênero" (Andersen, 2000, p. 70).

Considerando a influência que a sociedade exerce sobre o autoconceito e a auto-estima corporal das mulheres, este estudo procurou avaliar se o biotipo de atletas de alto nível desencadeia a aplicação de estereótipos sexuais por parte de grupos que não apresentam envolvimento desportivo, isto porque o biotipo destas foge dos padrões culturais. 
Segundo Giavoni (2002),

desportos com predomínio de características instrumentais (força, agressividade, violência) quando praticados por mulheres e desportos com predomínio de características expressivas (leveza, suavidade, delicadeza) quando praticados por homens, desencadeiam a aplicação de estereótipos sexuais. Isto porque as características do desporto versus o sexo do praticante contrariam a desejabilidade social, a qual se coaduna com as construções sociais de masculinidade e feminilidade (p. 28).

Além disso, procurou-se definir em quais dimensões da masculinidade e da feminilidade os grupos diferem entre si.

\section{Método}

\section{Amostra}

A amostra total foi composta por 90 estudantes com terceiro grau incompleto $(72,2 \%)$, sendo $56,66 \%$ do sexo feminino, com idade média de 25,12 anos ( $D P=6,38$ anos).

Os sujeitos foram classificados em três grupos de acordo com o envolvimento que apresentavam com o esporte. Assim, o grupo 1 foi formado por 27 atletas de ambos os sexos, sendo 55,55\% do sexo masculino, com idade média de 25,07 anos ( $D P=5,91$ anos) e nível de escolaridade igual a terceiro grau incompleto $(40,7 \%)$ e terceiro grau completo $(55,6 \%)$. Enquanto atletas, todos praticavam esporte variando a frequiência de três a cinco vezes por semana $(29,6 \%)$ e cinco a sete vezes por semana $(70,4 \%)$.

O grupo 2 foi formado por indivíduos envolvidos com o esporte. No caso foram escolhidos alunos e profissionais do curso de Educação Física da Universidade Católica de Brasília (UCB). A amostra foi composta por 41 indivíduos, sendo $51,22 \%$ do sexo feminino, com idade média de 25,61 anos $(D P=7,05$ anos $)$ e variando quanto ao nível de escolaridade de terceiro grau incompleto $(82,9 \%)$ a terceiro grau completo (12,20\%). Dos sujeitos entrevistados, 31,7\% praticavam atividade física de uma a três vezes por semana e $41,5 \%$ de três a cinco vezes por semana.

O grupo 3 foi formado por indivíduos que não apresentavam nenhum envolvimento com o esporte. A amostra foi composta por 22 estudantes universitários sedentários (90,9\%) de ambos os sexos, sendo $81,8 \%$ do sexo feminino, com idade média de 24,27 anos ( $D P=5,79$ anos).

\section{Instrumento}

O instrumento utilizado para avaliar os níveis de masculinidade e feminilidade atribuídos às atletas foi o Inventário dos Esquemas de Gênero do Autoconceito (IEGA) (Giavoni $\&$ Tamayo, 2000). Composto por 83 itens subdivididos em duas escalas - escala masculina (41 itens) e escala feminina (42 itens). Este instrumento avalia os esquemas de gênero presentes no autoconceito. O esquema masculino, avaliado a partir da escala masculina, é constituído por características pertinentes à masculinidade e, tal como este construto, encontra-se subdividido em quatro fatores principais denominados de: Negligência, Racionalidade, Ousadia e Agressividade e um fator de segunda ordem denominado de Indiferença. O esquema feminino, composto por características pertinentes à feminilidade, encontra-se subdividido em cinco fatores principais: Tolerância, Insegurança, Sensualidade, Emotividade e Responsabilidade e um fator de segunda ordem, o fator Sensibilidade.

Enquanto estruturas multidimensionais obtêm-se escores individuais e independentes para cada fator que compõe os esquemas masculino e feminino. A fim de se obter um escore resultante para cada um dos esquemas, utilizou-se o método de análises espaciais proposto por Giavoni e Tamayo (2003), na qual são encontradas as normas masculina ( $\mathrm{Nm}$ ) e feminina $(N f)$ das estruturas espaciais representativas dos esquemas masculino e feminino. Para tal análise, foram utilizadas as dimensões do esquema masculino (fatores $R a$ cionalidade, Indiferença e Ousadia) e do esquema feminino (fatores Insegurança, Sensualidade e Sensibilidade). Como os fatores de segunda ordem (Indiferença e Sensibilidade) são combinações subjacentes dos fatores de primeira ordem, estes não serão considerados na análise dos dados por tornar redundante a discussão, embora tenham sido utilizados no cálculo das normas masculina e feminina, respectivamente.

Em cada questionário foi anexada uma capa que apresentava uma colagem fotográfica de mulheres atletas de alto nível praticando diferentes modalidades desportivas (futebol, basquete, natação, pólo aquático, halterofilismo, corrida com barreira, corrida de longa distância, fisiculturismo e arremesso de peso).

\section{Procedimento}

Os questionários foram aplicados individualmente por um dos pesquisadores e/ou pelo treinador em se tratando dos grupos 1 e 3 e coletivamente, em sala de aula, com relação ao grupo 2. As instruções verbais dadas pelos aplicadores aos respondentes informavam que se tratava de um estudo sobre a percepção que os mesmos tinham a respeito da colagem fotográfica anexada à escala.

As instruções escritas do instrumento solicitavam que após observar a colagem durante alguns minutos, o respondente avaliasse o quanto cada item se aplicava às atletas, utilizando para isto de uma escala de cinco pontos no qual o escore 0 indicava que o item não se aplicava à atleta até $\mathrm{o}$ escore 4 indicando que o item se aplicava totalmente à atleta.

\section{Resultados}

Foi realizada uma análise exploratória inicial sobre os dados obtidos na amostra. Estas análises foram realizadas por grupo. Os casos faltosos (inferiores a 5\%) foram substituídos pelas médias obtidas para cada variável. O estudo da normalidade realizado sobre os fatores obtidos para as escalas masculina e feminina revelou os seguintes fatores com desvio de normalidade: fatores Insegurança e Responsabilidade no grupo 1 e os fatores Negligência e Agressividade no grupo 3. Foram detectados casos de outliers univariados nestes fatores e os seus valores discrepantes foram substituídos pelo valor extremo superior mais uma unidade, em se tratando de outliers acima da média e valores extremos inferiores menos uma unidade, em se tratando de outliers abaixo da 
média. Uma vez feitas estas correções, as variáveis atingiram índices normais.

Utilizando a variável "grupo" como variável independente e as variáveis Negligência, Racionalidade, Ousadia e Agressividade como variáveis dependentes foi realizada uma análise de variância multivariada (MANOVA) do tipo one way. Não foram encontradas diferenças significativas entre os três grupos estudados em relação aos fatores da escala masculina, Wilks' $\Lambda=0,93 ; \mathrm{F}(8,168)=0,74 ; p=0,66$.

Utilizando a variável "grupo" como variável independente e as variáveis Tolerância, Insegurança, Sensualidade, Emotividade e Responsabilidade como variáveis dependentes foi realizada uma análise de variância multivariada (MANOVA) do tipo One Way. Resultados significativos foram encontrados entre os grupos avaliados e as variáveis dependentes mensuradas, Wilks' $\Lambda=0,73 ; F(10,166)=2,86 ; p=0,003$.

As análises de variância univariadas (ANOVA) realizadas para cada variável dependente, testes estes conduzidos pela própria MANOVA ajustados pelo método de Bonferroni, revelaram que os fatores Sensualidade $(F(2,87)=6,68$; $p=0,002)$ e Responsabilidade $(F(2,87)=6,02 ; p=0,004)$ apresentavam diferenças significativas entre os grupos. Análises subseqüentes (post hoc) do tipo comparação para-par entre os grupos (pairwise comparisons, ajustadas pelo método de Bonferroni) demonstraram que em relação ao fator Sensualidade, o grupo $3(M=1,08 ; D P=0,19)$ percebe as mulheres atletas como sendo menos sensuais, atraentes, vaidosas, simpáticas e elegantes do que o grupo $1(M=2,0$, $D P=0,18)$ e grupo $2(M=1,80 ; D P=0,14)$.

Com relação ao fator Responsabilidade, os resultados demonstraram que o grupo $3(M=1,84 ; D P=0,12)$ as percebem como menos responsáveis, leais, discretas, cuidadosas, dedicadas, morais, caprichosas, fieis, íntegras, respeitosas, ajustadas e organizadas do que os grupos $1(M=2,39 ; D P=$ $0,11)$ e $2(M=2,29 ; D P=0,09)$.

A fim de se avaliar se os esquemas masculino e feminino variam entre si quanto ao nível de desenvolvimento, foram realizados testes $t$ pareados comparando-se as normas masculina $(\mathrm{Nm})$ e feminina $(\mathrm{N} f)$ obtidas para cada grupo. Foram encontrados resultados significativos entre as normas masculina e feminina dos grupos $3[t(21)=8,676$; $p=$

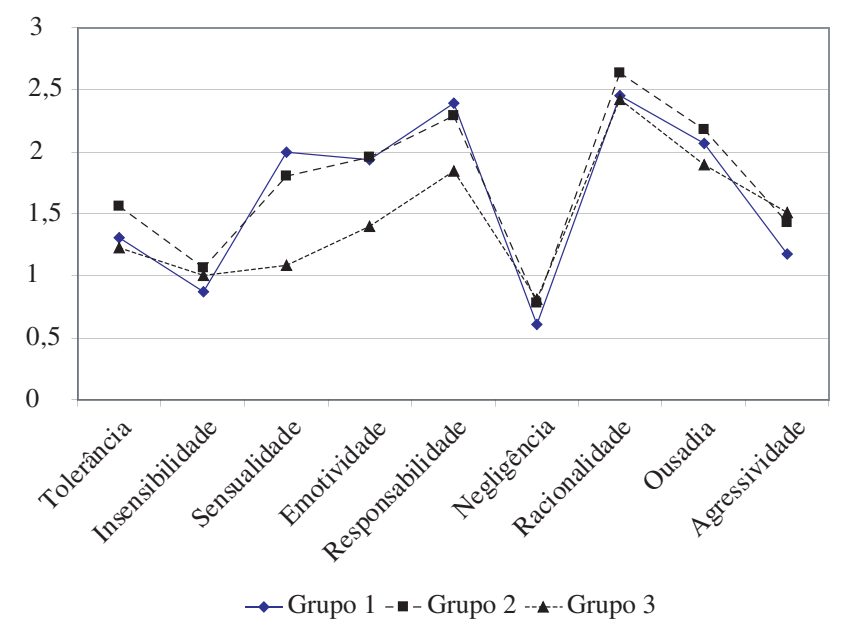

Figura 1. Médias das variáveis dependentes dos esquemas masculino e feminino.
$0,001]$ e 2 [t(40) = 3,106; $p=0,003]$, sendo que para ambos os grupos a norma masculina [grupo $3(\mathrm{Nm}=3,31 ; \mathrm{DP}=$ $0,8341)$ e grupo $2(N m=3,66 ; D P=0,857)]$ apresentaram valores superiores às normas femininas obtidas para ambos os grupos [grupo $3(N f=2,31 ; D P=0,22)$ e grupo $2(N f=$ $3,17 ; D P=0,16)]$, respectivamente. Em outras palavras, os grupos 3 e 2 perceberam as atletas como mais masculinas do que femininas, ao passo que para o grupo 1 não foram encontradas diferenças significativas $[t(26)=1,77 ; p=0,09]$ entre as normas masculina $(N m=3,38 ; D P=1,11)$ e feminina $(N f=3,145 ; D P=0,20)$. A figura 1 apresenta as médias obtidas para todas as variáveis dependentes analisadas, tanto do esquema masculino quanto do esquema feminino.

\section{Discussão}

Os resultados apresentam dados importantes que corroboram o estudo de Giavoni (2002) e comprovam a aplicação de estereótipos por parte de grupos que apresentam baixo envolvimento desportivo, no caso, o grupo 3. A falta de conhecimento e vivência na área desportiva propicia a objetivação de uma imagem distorcida das mulheres atletas, ao passo que os indivíduos que apresentam envolvimento, não as julgam utilizando os mesmos critérios. No caso do grupo 1 formado por indivíduos atletas de ambos os sexos, tomou-se o cuidado de avaliar se as percepções dos homens diferiam da auto-percepção das mulheres. Não foram encontradas diferenças significativas entre os dois grupos em nenhuma das variáveis analisadas anteriormente. Estes dados corroboram os estudos de Kunda (1999) nos quais o envolvimento e o nível de conhecimento sobre o grupo julgado encontram-se diretamente correlacionados com a aplicação de estereótipos.

Assim, observa-se que o grupo com baixo envolvimento desportivo tende a julgar as mulheres atletas com base em critérios definidos socialmente para a feminilidade, ou seja, critérios que estes indivíduos conhecem em maior ou menor intensidade por estarem inseridos em um meio sócio-cultural que estabelece normas, papéis, padrões de comportamentos e, inclusive, o biotipo desejável para homens e mulheres. Dos aspectos que compõem a feminilidade, os julgados como discrepantes - sensualidade e responsabilidade -, foram exatamente aqueles que a caracterizam no seu sentido mais nuclear e que são constantemente reforçados pelos meios de comunicação (Franzoi, 1995; Franzoi \& cols. 1989).

Segundo Giavoni e Tamayo (2000) o fator Sensualidade "focaliza a auto-imagem e sua influência na interação com os outros (...) e o fator responsabilidade avalia os princípios e valores que regem a conduta do self' (Giavoni \& Tamayo, 2000, p. 181). Uma análise mais criteriosa revelou que neste último fator, o grupo 3 diferiu dos demais grupos nos escores designados aos itens "discreta" e "ajustada", os quais foram mais baixos, confirmando novamente a influência da imagem das atletas sobre o julgamento destes indivíduos.

Sendo a feminilidade fortemente caracterizada pelos aspectos que compõem a sensualidade e que define o poder de atração de uma mulher, pode-se inferir que todo biotipo que fuja daquele estabelecido pela desejabilidade social como "biotipo feminino" tende a estimular a aplicação de estereótipos sexuais por parte daqueles que apresentem baixo 
envolvimento com o grupo estudado. Assim, as mulheres atletas tornam-se menos atraentes, sensuais, elegantes, emotivas, delicadas, amorosas e sensíveis devido ao biotipo que apresentam, biotipo este que traduz características definidas culturalmente como pertinentes à masculinidade, tais como: definição muscular, rigidez, força, potência, agressividade, determinação, entre outros.

Torna-se interessante ressaltar que os grupos não diferiram quanto aos componentes da masculinidade. Assim, apesar de perceberam o biotipo das atletas como divergente daquele definido culturalmente para as mulheres, o grupo 3 não as julgou como mais masculinas do que os demais grupos. A diferença do grupo 3 para os demais grupos reside especificamente nos fatores nucleares que caracterizam a feminilidade.

Entretanto, como o instrumento utilizado avalia os esquemas masculino e feminino presentes no autoconceito, efetuou-se uma última análise na qual os esquemas são tratados como um par. Esta análise objetiva avaliar se os esquemas são considerados como isométricos ou heterométricos, ou seja, se são proporcionais ou se há predominância de um esquema sobre o outro (Giavoni, 2000). A isometria, ou heterometria dos esquemas, é avaliada pelas normas masculina e feminina, que representam o nível de desenvolvimento dos esquemas cognitivos quando estes são avaliados como estruturas espaciais. De uma forma indireta, a predominância de um esquema sobre o outro revela a percepção dos grupos em relação à masculinidade e feminilidade atribuída às atletas.

Os resultados demonstraram que os grupos 3 e 2 julgaram as atletas como sendo portadoras de uma heterometria masculina, ou seja, o esquema masculino é maior do que o esquema feminino, ao passo que o grupo 1 as descreveu como isométricas, ou seja, não há diferença quanto ao nível de desenvolvimento dos dois esquemas. A diferença obtida entre os esquemas nos grupos 2 e 3 ocorreu, não porque o esquema masculino difere significativamente entre os grupos, mas porque o esquema feminino difere, tornando-se menor do grupo 2 para o grupo 3. Quanto menor o esquema feminino, mais heterométrico torna-se o par de esquemas estudado. Assim, pode-se dizer que os grupos 2 e 3 julgaram as atletas como sendo mais masculinas do que femininas, ao passo que o grupo 1 as julgou como portadoras de um equilíbrio entre a masculinidade e a feminilidade.

Conclui-se, portanto, que o biotipo de mulheres atletas vinculadas a desportos com características masculinas desencadeiam a aplicação de estereótipos sexuais por parte de grupos que ignoram o atleta e o ambiente desportivo. Possivelmente, este viés perceptivo somado à pressão social de assumir características, papéis, atitudes, comportamentos e, até o biotipo condizente com os padrões culturais estabelecidos para a feminilidade, acabem por exercer forte influência sobre meninas e adolescentes que estejam na fase de transição entre a aprendizagem e aperfeiçoamento de um determinado desporto e/ou treinamento. Talvez, esta seja uma das causas do abandono desportivo (drop out) tão freqüente entre adolescentes em início de carreira atlética.

Outro fator importante a ser considerado em estudos futuros refere-se à formação de grupos de acordo com os níveis de masculinidade e feminilidade que os indivíduos apresentem e, após esta classificação, avaliar o julgamento destes em relação aos atletas. Isto porque, possivelmente, o predomínio de um esquema sobre o outro resultará em julgamentos distintos de um mesmo estímulo. Homens e mulheres com predomínio do esquema masculino sobre o feminino (heteroesquemáticos masculinos) tenderão a julgar estímulos masculinos diferentemente de homens e mulheres com predomínio do esquema feminino (heteroesquemáticos femininos) e homens e mulheres com equilíbrio entre os esquemas (isoesquemáticos).

Outro estudo relevante seria avaliar as percepções de diferentes grupos em relação a colagens de desportistas masculinos praticantes de desportos com características femininas, ou seja, o inverso do presente estudo.

\section{Referências}

Andersen, M. B. (2000). Doing sport psychology. Lower Mitchan: Human Kinetics

Antill, J. K. \& Russell, G. (1982). The factor structure of the Bem Sex-Role Inventory: Method and sample comparisons. Australian Journal of Psychology, 34(2), 183-193.

Archer, J. (1989). The relationship between gender-role measures: A review. British Journal of Social Psychology. 28(2), 173-184.

Aubé, J. \& Koestner, R. (1992). Gender characteristics and adjustment: A longitudinal study. Journal of Personality and Social Psychology, 63(3), 485-493.

Barberá, E. (1998). Psicología del género. Barcelona: Editorial Ariel.

Bem, S. L. (1974). The measurement of psychological androgyny. Journal of Consulting and Clinical Psychology, 42(2), 155162.

Bem, S. L. (1981). Gender schema theory: A cognitive account of sex typing. Psychological Review, 88(4), 354-364.

Bernard, L. C. (1981). The multidimensional aspects of masculinityfemininity. Journal of Personality and Social Psychology, 41(5), 797-802.

Constantinople, A. (1973). Masculinity - femininity: An exception to a famous dictum. Psychological Bulletin, 80(5), 389-407.

Feather, N. T. (1978). Factor structure of the Bem Sex-Role Inventory: Implications for the study of masculinity, femininity, and androgyny. Australian Journal of Personality, 30(2), 241-254.

Ferreira, A. B. H. (1999). Novo Aurélio Século XXI: O dicionário da língua portuguesa. Rio de Janeiro: Nova Fronteira.

Franzoi, S. L. (1995). The body-as-object versus the body-asprocess: Gender differences and gender considerations. Sex Roles, 33(5-6), 417-437.

Franzoi, S. L. \& Shields, S. A. (1984). The body esteem scale: Multidimensional structure and sex differences in a college population. Journal of Personality Assessment, 48(2), 173178.

Franzoi, S. L., Kessenich, J. J. \& Surgrue, P. A. (1989). Gender differences in the experience of body awareness: An experiential sampling study. Sex Roles, 21(7-8), 499-515.

Gaa, J. P., Liberman, D. \& Edwards, T. A. (1979). A comparative factor analysis of the Bem Sex Role Inventory and the Personality Attributes Questionaire. Journal of Clinical Psychology, 35(3), 592-598.

Gaudreau, P. (1977). Factor analysis of the Bem Sex-Role Inventory. Journal of Consulting and Clinical Psychology, 45(2), 299302. 
Giavoni, A. (2000) A Interação entre os Esquemas Masculino e Feminino do Autoconceito: Modelo Interativo. Tese de doutorado, Universidade de Brasília, Brasília.

Giavoni, A. (2002). Estereótipos sexuais aplicados a nadadoras. Revista Brasileira Ciência e Movimento, 10(2), 27-32.

Giavoni, A. \& Tamayo, A. (2000). Inventário dos Esquemas de Gênero do Autoconceito (IEGA). Psicologia: Teoria e Pesquisa, 16(2), 175-184.

Giavoni, A. \& Tamayo, A. (2003). Análise espacial: conceito, método e aplicabilidade. Psicologia: Reflexão e Crítica, 16 (2), 303-307.

Koestner, R. \& Aubé, J. (1995). A multifactorial approach to the study of gender characteristics. Journal of Personality, 63(3), 681-710.
Kunda, Z. (1999). Social cognition. Marketing sense of people. Cambridge: MIT Press.

Markus, H. (1977). Self-information and processing information about the self. Journal of Personality and Social Psychology, 35(2), 63-78.

Markus, H., Crane, M., Bernstein, S. \& Siladi, M. (1982). Selfschemas and gender. Journal of Personality and Social Psychology, 42, 38-50.

Rodrigues, A., Assmar, E. \& Jablonski, B. (1999) Psicologia social. Petrópolis: Vozes.

Spence, J. T., Helmreich, R. \& Stapp, J. (1975). Ratings of self and peers on sex role attributes and their relation to self-esteem and conceptions of masculinity and femininity. Journal of Personality and Social Psychology, 32(1), 29-39.

Recebido em 05.11.2003

Primeira decisão editorial em 27.10.2004

Versão final em 29.10.2004

Aceito em 03.11.2004 